\title{
e-ISSN $1983-0572$ \\ Ocorrência do Grilo Endecous em Quatro Cavernas no Município de Uruará - PA, Brasil
}

\author{
Reinaldo Lucas Cajaiba
}

Universidade de Trás-os-Montes e Alto Douro, e-mail: reinaldocajaiba@hotmail.com.

$$
\text { EntomoBrasilis } 5 \text { (2): 12O-124 (2012) }
$$

Resumo. O gênero Endecous (Ensifera: Phalangopsidae) pode ser encontrado em toda região neotropical. Cinco espécie desse gênero está presente no Brasil: Endecous arachnopis Saussure, Endecous cavernicolus Costa Lima, Endecous itatibensis Rehn, Endecous abbreviatus Piza e Endecous betariensis de Mello \& Pellegatti-Franco. Este trabalho teve como objetivo analisar a abundância de Endecous em quatro cavernas do município de Uruará-PA. As coletas foram realizadas no período de agosto a novembro de 2011 com armadilhas pitfall sem isca. Foram coletados 832 espécimes. Como resultados foram obtidos valores significativos para a baixa temperatura $\left(R^{2}=0,9\right)$.

Palavras-chave: Bioindicadores; Invertebrados; Preservação Ambiental

\section{Occurrence of the Cricket of the Genus Endecous in Four Caves of the City of Uruará, State of Pará, Brazil.}

Abstract. The genus Endecous (Ensifera: Phalangopsidae) can be found in the entire neotropical region. Five species of this genus occur in Brazil: Endecous arachnopis Saussure, Endecous cavernicolus Costa Lima, Endecous itatibensis Rehn, Endecous abbreviatus Piza and Endecous betariensis de Mello \& Pellegatti-Franco. The present study was aimed to analyze the abundance of crickets of the genus Endecous in four caves in the city of Uruará-PA. The collections were performed from August to November 2011, using pitfall without bait. 832 specimens were collected. Significant values were obtained for the low temperature $\left(\mathrm{R}^{2}=0.9\right)$.

Keywords: Bioindicators; Environmental Preservation; Invertebrates

A s cavernas podem ser definidas como cavidades naturais subterrâneas, sendo estas, espaços que geralmente possuem continuidade com ambientes epígeos e suscetíveis à colonização por espécies preadaptadas (GILBERT et al. 1994). Tais ambientes possuem características singulares que os diferenciam dos ecossistemas epígeos. De maneira geral, são caracterizadas por uma elevada estabilidade ambiental e pela ausência permanente de luz (Poulson \& White 1969; Culver 1982).

No meio hipógeo, as fezes de morcegos e grilos e os regurgites de corujas podem formar grandes acúmulos de matéria orgânica em decomposição constituindo fonte essencial de energia (GNASPININETO 1989) já que os organismos fotoautótrofos são incapazes de se desenvolverem nesse ambiente.

Portanto, a distribuição espacial da fauna cavernícola pode ser determinada por inúmeras variáveis, dentre elas os recursos alimentares (Poulson \& Culver 1969).

As cavernas formam ambientes únicos, com uma fauna peculiar, formando ilhas e/ou refúgios no local onde estão inseridas, sendo consideradas sítios de especial interesse científico. Podem apresentar uma fauna frágil e vulnerável aos impactos causados no ambiente físico por interferências de ações humanas ou fenômenos naturais (Alves 2007). Os animais que ai habitam são limitados em suas capacidades de dispersão, devido à descontinuidade no habitat apropriado, como consequência, eles apresentam altos níveis de endemismos (CHRISTMAn et al. 2005).

Apesar de o Brasil possuir inúmeras cavernas, foi a partir da década de 80 que o conhecimento sobre a fauna cavernícola brasileira teve maior divulgação (DEsSEN et al. 1980; CHAÜNOWICZ
1986; Godoy 1986; Trajano \& Gnaspini 1986; Trajano 1987; Trajano \& Moreira 1991; Gnaspini \& Trajano 1994; Pinto-DaROCHA 1994; 1995; BAHIA \& FERREIRA 2005).

O conhecimento da entomofauna de âmbito regional tem grande importância para a obtenção de informações a respeito do comportamento das espécies, e a relação existente entre inseto, ambiente e ação antrópica.

Os insetos são ótimas ferramentas em estudos de ecologia dos ecossistemas naturais como bioindicadores de perturbação ambiental(RosENBERG etal.1986), pois sãoaltamenteinfluenciados pelas mudanças do hábitat (THOMAZINI \& THOMAZINI 2000; RibAs et al. 2003).

Dentre os insetos, os grilos são importantes ecologicamente como fontes de alimento para outros animais, e como bioindicadores, especialmente porque respondem de forma localizada a impactos (Hoffmann et al. 2002) e a perturbações ambientais (SPERBER et al. 2007). Através dessas respostas é possível identificar locais impactados de forma precisa, pois a riqueza de espécies pode apresentar uma resposta positiva ao incremento da disponibilidade e heterogeneidade de recursos, complexidade estrutural, heterogeneidade espacial e mudança de condições (Bustos \& UlLOA-CHACón 1997; VASCONCELOS 1999).

O gênero Endecous pode ser encontrado em toda região neotropical. É composto por dez espécies (Отте et al. 2005). Cinco delas presente no Brasil: Endecous arachnopis Saussure, Endecous cavernicolus Costa Lima, Endecous itatibensis Rehn, Endecous abbreviatus Piza, e Endecous betariensis de Mello \& Pellegatti-Franco; uma no México: Endecous annulatus Bilimek; uma no Uruguai: Endecous onthophagus Berg; uma na Bolívia: 
Endecous ferruginosus Bruner; e duas na Argentina: Endecous lizeri Rehn e Endecous hubbelli Lieberman.

Esse trabalho objetivou fazer o levantamento da presença do grilo Endecous (Ensifera: Phalangopsidae) em quatro cavernas do município de Uruará-PA.

\section{MATERIAL E MÉTODOS}

Local de estudo. O município de Uruará está localizado no estado do Pará, ao longo da Rodovia Transamazônica, mais precisamente no $\mathrm{km} \mathrm{180}$, no trecho entre os municípios de Altamira e Itaituba.

O clima na região apresenta um caráter bimodal de precipitação, com uma época seca ou de estiagem e outra chuvosa.

O clima local, segundo a classificação de Köppen é do tipo Awi, típico de clima quente úmido, com média do total pluviométrico anual da ordem de $2000 \mathrm{~mm}$, cujo período chuvoso vai de dezembro a maio e, o seco de junho a novembro. A temperatura média anual varia de 25 a $28^{\circ} \mathrm{C}$ e umidade relativa do ar fica acima de $80 \%$ (BASTOS et al. 1984).

O estudo foi desenvolvido em quatro cavernas. Como as cavernas ainda não estão cadastradas no Cadastro Nacional de Cavernas$\mathrm{CNC}$, as mesmas receberão as nomenclaturas de CAV1 a CAV4 e sua localização geográfica.

* CAV1-Caverna localizada no km 175 sul: coordenadas geográfica $\mathrm{So}^{\circ}{ }^{\circ} 48,128^{\prime}$ 'W $53^{\circ} 40,584^{\prime}$. Sua extensão é de aproximadamente $160 \mathrm{~m}$. Apresenta alto grau de ação antropogênica. Está localizada no meio de pastagens, não existindo vegetação em sua proximidade.

* CAV2-Caverna localizada no km 201 sul: coordenadas geográfica $\mathrm{So}^{\circ}{ }^{\circ} 55,877^{\prime}$ Wo $53^{\circ} 53,226$ '. Sua extensão é de aproximadamente 165 m. A caverna apresenta um alto grau de perturbação antropogênica, estando a mesma localizada no centro de uma fazenda de criação bovina, onde foi possível constatar várias fezes desses animais em sua entrada. Como há uma rodovia a cerca de $100 \mathrm{~m}$ ao lado oeste, a mesma é frequentemente visitada por turistas.

* CAV3-Caverna localizada no km 165 sul: So3 ${ }^{\circ} 46,470^{\prime}$ Wo5 $3^{\circ} 35,451^{\prime}$. Sua extensão é de aproximadamente $270 \mathrm{~m}$. A caverna está localizada em uma região de difícil acesso, sendo pouca visitada. Apresenta alto grau de preservação, sendo cercada por densas florestas. Estende-se no sentido oeste, apresentando água em todo seu percurso. A quantidade de morcegos observada nessa caverna é altíssima.

* CAV4-Caverna km 195 sul: So3 ${ }^{\circ} 54,774 ’$ W53 ${ }^{\circ} 49,829$ '. Sua extensão é de aproximadamente $330 \mathrm{~m}$, sendo a maior caverna estudada neste trabalho. Estende-se no sentido oeste. A água está distribuída por quase todo percurso. Destaca-se pelo alto grau de preservação da vegetação ao entorno. Porém, é necessário medidas de conscientização e uma ação efetiva do poder público, pois no momento das coletas havia iniciado um desmatamento em áreas bem próxima para plantio de pastagem.

Coleta de Dados. O presente estudo contou com sua fase de campo realizada entre os meses de Agosto/2011 a Novembro/2011.

Para a coleta de Endecous foram utilizadas armadilha do tipo "Pitfall" sem atrativos, sendo instaladas cinco armadilhas em cada caverna.

O tempo decorrido da montagem das armadilhas em cada caverna até a sua desmontagem foi de quatro dias. Durante a semana em que as armadilhas estiveram montadas, houve monitoramento a cada dois dias para verificar as condições das armadilhas. A triagem, identificação e o trabalho com os dados, foram realizados no período entre cada coleta, através de um estereomicroscópio com lente de aumento 20x.

As armadilhas foram instaladas desde a entrada da caverna e distribuídas por todo seu percurso. Em cada ponto foi medida a temperatura para com isso correlacionar a abundância dos ortópteros com esse fator.

Tratamentos estatísticos. Foi aplicado o teste de similaridade de Bray-Curtis para analisar se há semelhança entre os ambientes estudados. Para analisar a relação entre temperatura e abundância de ortópteros foi calculado o $\mathrm{R}^{2}$. Essas análises foram feitas com o programa PAST (HAMMER et al. 2003).

\section{RESULTADOS E DISCUSSÃO}

Nas quatro cavernas estudadas foram encontradas 832 indivíduos. A caverna mais abundante foi a $\mathrm{CAV}_{3}$ com 321 (39\%), seguido pelas CAV4 com 261 (31\%) e CAV2 com 188 (23\%). A que teve a menor representatividade foi a CAV1 com 62 (7\%) espécimes (Figura 1).

Observou-se que a maior abundância desses animais provavelmente está relacionada com a melhor preservação das cavernas, sendo que a $\mathrm{CAV}_{3}$ a mais preservada, apresentou uma maior quantidade de espécimes. Outro fator que também poderá ter influenciado na abundância da $\mathrm{CAV}_{3}$ e CAV4 é a presença da água em todo percurso e também a grande quantidade de morcegos observados nesses ambientes, uma vez que as fezes dos morcegos são uma grande fonte de alimentos em ambientes cavernícolas.

Segundo Trajano \& GnaSpini (1991) os grilos Endecous spp. estão efetivamente entre os macroinvertebrados onívoros/detritívoros mais comuns e de ampla ocorrência nas cavernas brasileiras.

Em vários outros levantamentos faunísticos em ambientes cavernícolas foram encontrados a presença desses grilos (Gnaspini \& Trajano 1994; Pinto-da-Rocha 1995; Alves 2007; BAHIA 2007), mas até o momento nenhum trabalho foi realizado especificamente para medir a análise faunística de Orthoptera para o estado do Pará, impossibilitando qualquer comparação dos resultados obtidos.

Endecous são trogófilos que podem ser encontrados desde a zona de entrada, até áreas mais profunda, variando sua distribuição hipógea nas diferentes regiões (PINTO-DA-Rocha 1995), não apresentando preferências nítidas, sendo observados em diferentes locais das cavernas. Essas observações confirmam a interrelação entre estratificação e biologia alimentar (TRAJANo 1991).

Pelos dados obtidos nessa pesquisa a baixa temperatura também foi um fator que influenciou positivamente na abundância desses animais nas cavernas $\left(\mathrm{R}^{2}=0,9\right)$ Figura 2, sendo dessa forma um ótimo indicador ecológico.

Muitos autores vem utilizando os grilos como organismos modelo para estudos biológicos, em fisiologia (HoEnen \& MARques 1998), bioquímica e biologia molecular (AvERY 2002; NACHMAN et al. 2004), evolução (BAILEY 2003; EBERHARD 2004). São ainda utilizados para comportamento territorial e reprodutivo (BATEMAN \& Ferguson 2004) e também da ecologia (SPERBER et al. 2003; RiBAs et al. 2005).

A análise de similaridade comprovou similaridade moderada entre as $\mathrm{CAV}_{3}$ e CAV4 (similaridade de 53\%) Figura 3. Enquanto que a CAV1, em virtude de suas condições peculiares, foi a que mais se distanciou no agrupamento. Esta gruta apresentou os menores valores de similaridade em relação às outras grutas estudadas.

Baseado nos resultados obtidos, podemos concluir que mesmo as cavernas geograficamente próximas, a similaridade poderá 


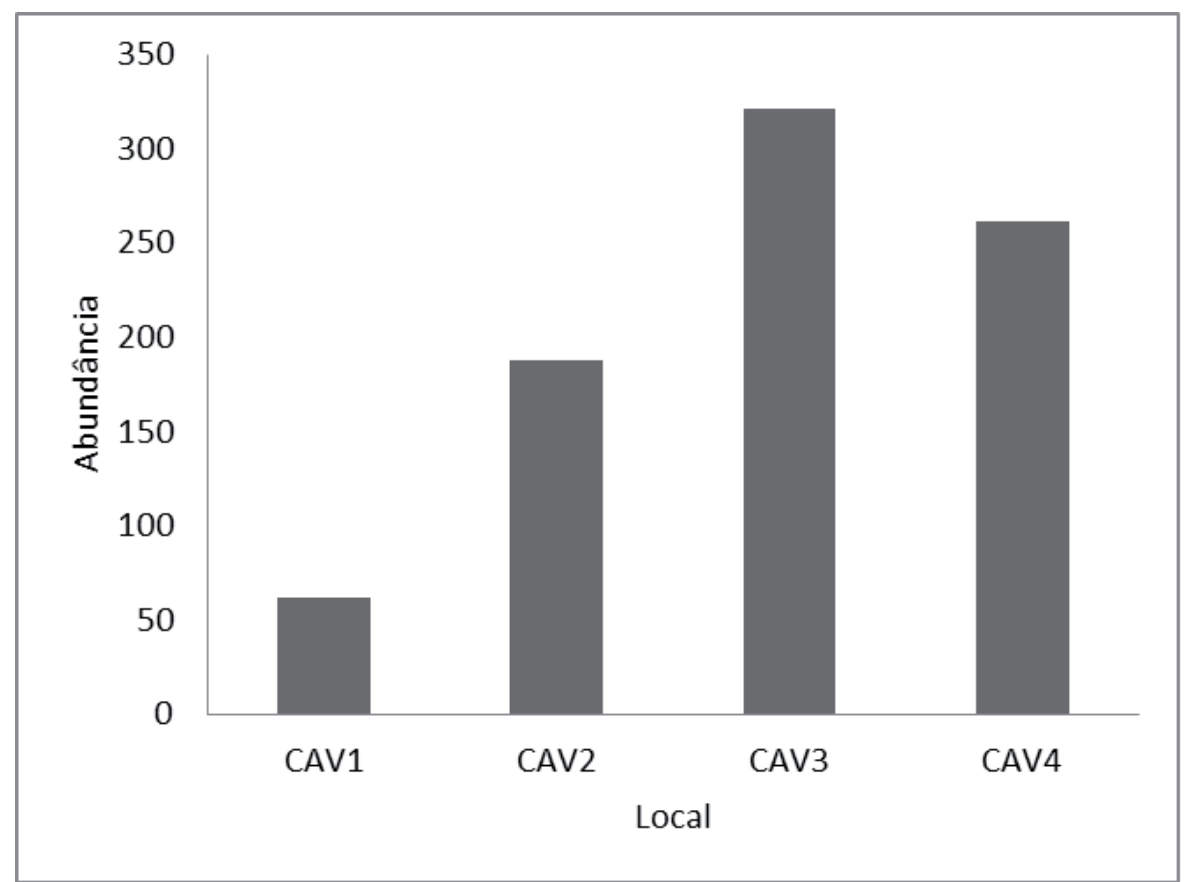

Figura 1. Abundância de Endecous spp. observados em quatro cavernas do município de Uruará, PA.

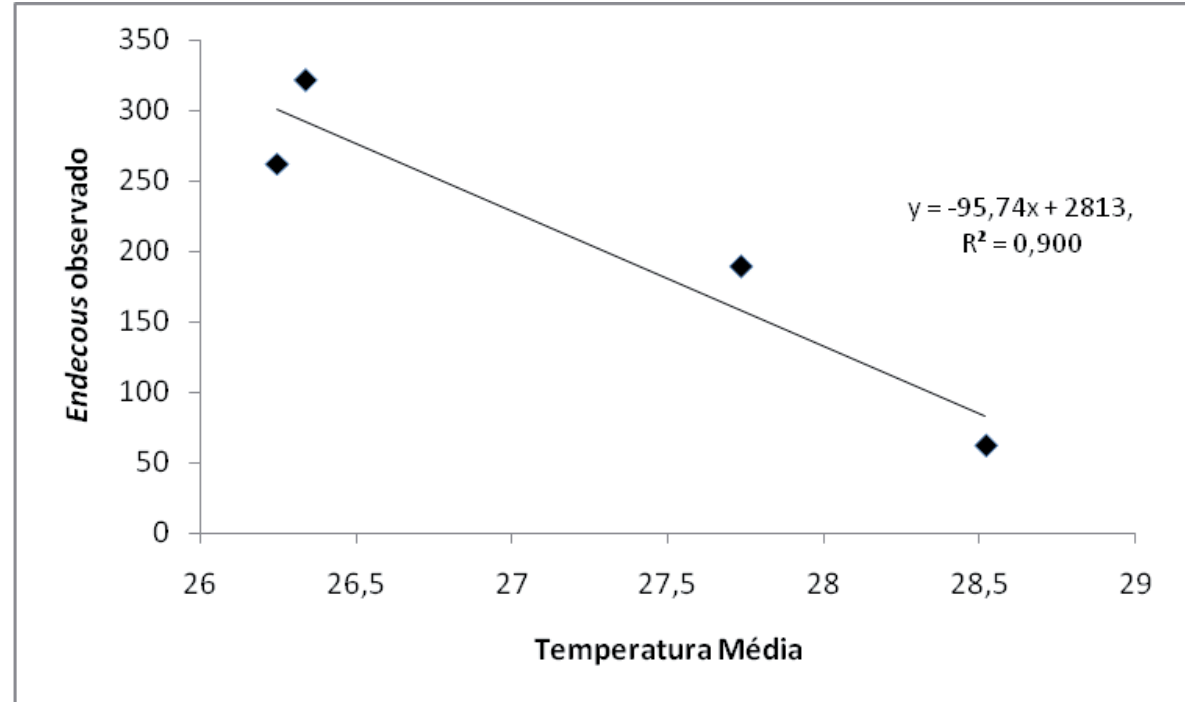

Figura 2. Relação entre temperatura média das cavernas com a abundância de Endecous spp. capturados em quatro cavernas no município de Uruará, PA.

ser baixa, tendo em vista que as condições biológicas e físicoquímicas de cada ambiente interferem na diversidade $\mathrm{e}$ dominância das comunidades. As distribuições da diversidade cavernícola é altamente heterogênea, variando de caverna para caverna e até mesmo dentro de uma mesma caverna (Prous et al. 2004; FERREIRA 2005).

São conhecidos aproximadamente 3.000 espécies de grilos (ОттЕ et al. 2005), mas estima-se que ainda exista mais que o triplo a ser descoberto pela ciência. Por isso a importância de se conhecer com mais detalhe esse taxa nas mais diversas condições, havendo assim a necessidade de estudos aprimorados.

O município de Uruará está sofrendo grandes perdas em sua vegetação original causados por madeireiros e pecuaristas. Por tais motivos, torna-se extremamente urgente um estudo minuncioso, através do monitoramento da fauna subterrânea na região, uma vez que foi demonstrado nesse estudo que ambientes alterados apresentam menor abundância.

A urgência de se promover eventos e palestras que abordem temas sobre educação ambiental é de extrema necessidade com o objetivo maior de sensibilizar as comunidades próximas e o público visitante. Além disso, preservar a biodiversidade tanto do meio cavernícola como do meio externo é uma medida fundamental para manutenção da paisagem subterrânea contribuindo para futuros estudos.

\section{Bray-Curtis Cluster}

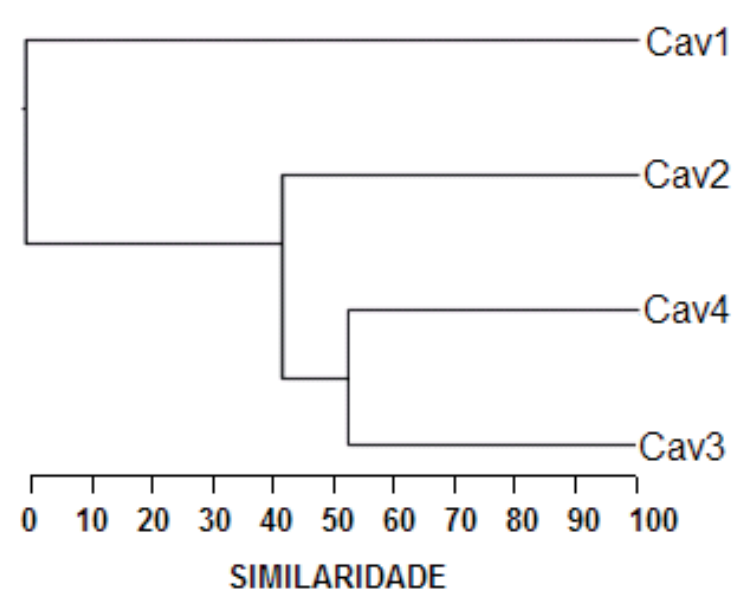

Figura 3. Análise de agrupamento. Dados de abundância de Endecous spp., amostrados em quatro cavernas no município de Uruará, PA. 


\section{AGRADECIMENTOS}

O autor agradece a suma importância na realização deste trabalho da ONG Brucutu que gentilmente cedeu os biólogos Nilson Chaves e Kael Rodrigo e o Prof. Wilson que nos acompanharam em todas as cavernas.

\section{REFERÊNCIAS}

Alves, V.R., 2007. Artrópodes cavernícolas com ênfase em flebotomíneos (Diptera: Psychodidae) do município de Presidente Figueiredo, AM. Dissertação (Mestrado em Ciências Biológicas) - Instituto Nacional de Pesquisas da Amazônia/Universidade Federal do Amazonas, Manaus, 82 p.

Avery, P.J., 2002. Fitting interconnected Markov chain modelsDNA sequences and test cricket matches. Journal of the Royal Statistical Society Series D-The Statistican, London, 51: 267278.

Bahia, G. R. \& R. L. Ferreira, 2005. Influência das características físicoquímicas e da matéria orgânica de depósitos recentes de guano de morcego na riqueza e diversidade de invertebrados de uma caverna calcária. Revista Brasileira de Zoociências, 7: 165-180.

Bahia, G.R., 2007. Sucessão ecológica em guano de morcegos insetívoros em cavernas. Dissertação (Mestrado em Ecologia, Conservação e Manejo da Vida Silvestre) - Universidade Federal de Minas Gerais, Belo Horizonte, 107 p.

Bailey, W.J., 2003. Insect duets: underlying mechanisms and their evolution. Physiological Entomology, London, 28: 157174.

Bastos, T.X., E. J. Da Rocha, P.A. Rolim, T.D. Diniz, E.C. Dos Santos, R.A. Nobre, E.M. Cutrim \& L.L. Mendonça, 1984. $\mathrm{O}$ estudo atual dos conhecimentos de clima da Amazônia brasileira com finalidade agrícola. Belém EMBRAPA, CPATU Doc. n. 36.

Bateman, P.W. \& J.W.H. Ferguson, 2004. Male choice in the Botswana armoured ground cricket Acanthoplus discoidalis (Orthoptera: Tettigoniidae: Hetrodinae). Can and how, do males judge female mating history? Journal of Zoology, 262: 305-309.

Bustos, J.H. \& P. Ulloa-Chacón, 1997. Mirmecofauna y perturbación en um bosque de neblina neotropical (Reserva Natural Hato Viejo, Valle del Cauca, Colombia). Revista de Biologia Tropical, 44: 259-266.

Chaünowicz, F., 1986. Observações preliminares sobre o ecossistema da gruta Olhos D'Âgua, Itacarambi-MG. Espeleo-Tema, 15: 67-79.

Christman, M.C., D.C. Culver, M.K. Madden \& D. White, 2005. Patterns of endemism of the eastern North American caver fauna. Journal of Biogeopraphy, 32: 1441-1452.

Culver, D.C., 1982. Cave life: Evolution and Ecology. Cambridge: Harvard Univ. Press, 189p.

Dessen, E.M.B., V.R. Eston, M.S. Silva, M.T.T. Beck \& E. Trajano, 1980. Levantamento preliminar da fauna de cavernas de algumas regiões do Brasil. Ciência e Cultura, 32: 714-725.

Eberhard, W.G., 2004. Male-female conflict and genitalia: failure to confirm predictions in insects and spiders. Biological Reviews, 79: 121-186.

Ferreira, R.L., 2005. A vida subterrânea nos campos ferruginosos. O Carste, 17: 106-115.

Gilbert, J., D.L. Danielpol \& J.A. Stanford, 1994. Groundwater Ecology. New York: Academic Press, 571 p.

Gnaspini, P. \& E. Trajano, 1994. Brazilian cave invertebrates, with a checklist of troglomorphic taxa. Revista Brasileira de Zoologia, 38: 549-584.

Gnaspini-Neto, P., 1989. Análise comparativa da fauna associada a depósitos de guano de morcegos cavernícolas no Brasil. Primeira aproximação. Revista Brasileira de Entomologia, 33: 183-192.

Godoy, N.M., 1986. Nota sobre a fauna cavernícola de BonitoMS. Espeleo-Tema, 15: 80-92.
Hammer, Ø., D.A.T. Harper \& P.D. Ryan, 2003. Past Palaentological Statistics, ver. 1.12. Disponível em: $<$ http://folk.uio.no/ohammer/past>. Acesso em $15 \mathrm{de}$ outubro de 2011.

Hoenen, S. \& M. D. Marques, 1998. Circadian patterns of migration of Strinatia brevipennis (Orthoptera: Phalangopsidae) inside a cave. Biological Rhythm Research, 29: 480-487.

Hoffmann, B.D., L.M. Lowe \& A.D. Griffiths, 2002. Reduction in cricket (Orthoptera: Ensifera) populations along a gradient of sulphur dioxide from mining emissions in northern Australia. Australian Journal of Entomology, 41: 182-186.

Nachman, R.J., G.M. Coast, K. Kaczmareck, H.J. Williams \& J. Zabrocki, 2004. Stereochemistry of insect kinin tetrazole analogues and their diuretic activity in crickets. Acta Biochimica Polonica, 51: 121-127.

Otte, D., D.C. Eades \& P. Naskrecki, 2005. Orthoptera species file online. Version 2.2. Disponível em: <http://osf2.orthoptera.org >. Acesso em o5 agosto de 2011.

Pinto-Da-Rocha, R., 1994. Invertebrados cavernícolas da porção meridional da Província Espeleológica do Vale do Ribeira, sul do Brasil. Revista Brasileira de Zoologia, 10: 229-255.

Pinto-Da-Rocha, R., 1995. Sinopse da fauna cavernícola do Brasil (1907-1994). Papéis Avulsos de Zoologia, 39: 61-173.

Poulson, T.L. \& D.C. Culver, 1969. Diversity in terrestrial cave communities. Ecology, 50: 153-157.

Poulson, T.L. \& W.B. White, 1969. The cave environment. Science, 165: 971-980.

Prous, X., R.L. Ferreira \& R.P. Martins, 2004. Ecotone delimitation: epigean hypogean transition in cave ecosystems. Austral Ecology, 29: 374382.

Ribas, C.R., J.H. Schoereder, M. Pic \& S.M. Soares, 2003. Tree heterogeneity, resource availability, and larger scale processes regulating arboreal ant species richness. Austral Ecology, 28: 305-314.

Ribas, C.R., T.G. Sobrinho, J.H. Schoereder, C.F. Sperber, \& C. Lopes-Andrade, 2005. How large is large enough for insects? Forest fragmentation effects at three spatial scales. Acta Oecologica, 27: 31-41.

Rosenberg, D.M., H.V Danks \& D.M. Lehmkuhl, 1986. Importance of insects in environmental impact assessment. Environmental Management, 10: 773-783.

Sperber, C.F., G.H. Vieira \& M.H. Mendes, 2003. Aprimoramento da amostragem de grilos de serrapilheira (Orthoptera: Gryllidae) por armadilha. Neotropical Entomology, 32: 733735 .

Sperber, C.F., L.G.S. Soares \& M.R. Pereira, 2007. Litter disturbance and trap spatial positioning affects number of captured individuals and genera of crickets (Orthoptera: Grylloidea). Journal of Orthoptera Research, 16: 1-7.

Thomazini, M.J. \& A.P.B.W. Thomazini, 2000. A fragmentação florestal e a diversidade de insetos nas florestas tropicais úmidas. Rio Branco - Acre: Embrapa. Documentos, 57.

Trajano, E. \& J.R. Moreira, 1991. Estudo da fauna de cavernas da província espeleológica arenítica Altamira-Itaituba, Pará. Revista Brasileira de Biologia, 51: 13-29.

Trajano, E. \& P. Gnaspini, 1986. Observações sobre a mesofauna cavernícola do Alto Vale do Ribeira-SP. Espeleo-Tema, 15: 28-32.

Trajano, E. \& P. Gnaspini, 1991. Composição da fauna cavernícola brasileira, com uma análise preliminar da distribuição dos táxons. Revista Brasileira Zoologia, 7: 383-407.

Trajano, E., 1987. Fauna cavernícola brasileira: composição e caracterização preliminar. Revista Brasileira Zoologia, 3: 533-561.

Trajano, E., 1991. Population ecology of Pimelodella kronei, troglobitic catfish from Southeastern Brazil (Siluriformes, Pimelodidae). Enviromental Biology of Fishes, 30: 407-421.

Vasconcelos, H.L., 1999. Effects of forest disturbance on the structure of ground foraging ant communities in central Amazonia. Biodiversity and Conservation, 8: 409-420. 
Recebido em: 02/01/2012

Aceito em: 09/04/2012

Como citar este artigo:

Cajaiba, R.L., 2012. Ocorrência do Grilo Endecous em Quatro Cavernas no Município de Uruará - PA, Brasil. EntomoBrasilis, 5(2): $120-124$. Acessível em: http://www.periodico.ebras.bio.br/ojs/index.php/ebras/article/view/217
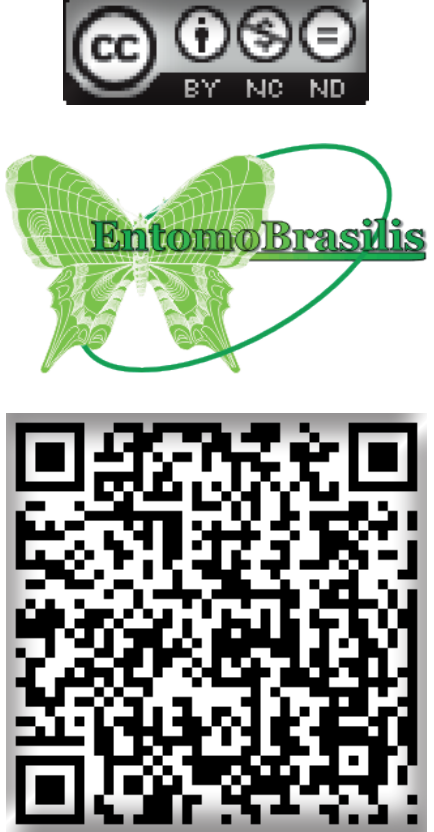